\title{
OPEN Reusable surface amplified nanobiosensor for the sub PFU/mL level detection of airborne virus
}

\author{
Junghyun Shin ${ }^{1}$, Hyeong Rae Kim ${ }^{3}$, Pan Kee Bae ${ }^{5}$, Haneul Yoo ${ }^{1}$, Jeongsu Kim¹ ${ }^{1}$, Yoonji Choi ${ }^{1}$, \\ Aeyeon Kang ${ }^{8}$, Wan S. Yun ${ }^{8}$, Yong Beom Shin ${ }^{5,6,7}$, Jungho Hwang ${ }^{4}$ \& Seunghun Hong ${ }^{2 \bowtie}$
}

We developed a reusable surface-amplified nanobiosensor for monitoring airborne viruses with a sub-PFU/mL level detection limit. Here, sandwich structures consisted of magnetic particles functionalized with antibodies, target viruses, and alkaline phosphatases (ALPs) were formed, and they were magnetically concentrated on $\mathrm{Ni}$ patterns near an electrochemical sensor transducer. Then, the electrical signals from electrochemical markers generated by ALPs were measured with the sensor transducer, enabling highly-sensitive virus detection. The sandwich structures in the used sensor chip could be removed by applying an external magnetic field, and we could reuse the sensor transducer chip. As a proof of concepts, the repeated detection of airborne influenza virus using a single sensor chip was demonstrated with a detection limit down to a sub-PFU/mL level. Using a single reusable sensor transducer chip, the hemagglutinin (HA) of influenza $A$ (H1N1) virus with different concentrations were measured down to 10 aM level. Importantly, our sensor chip exhibited reliable sensing signals even after more than 18 times of the repeated $H A$ sensing measurements. Furthermore, airborne influenza viruses collected from the air could be measured down to 0.01 PFU/ $\mathrm{mL}$ level. Interestingly, the detailed quantitative analysis of the measurement results revealed the degradation of HA proteins on the viruses after the air exposure. Considering the ultrasensitivity and reusability of our sensors, it can provide a powerful tool to help preventing epidemics by airborne pathogens in the future.

Respiratory viruses such as influenza and corona can spread out through the air and cause significant health problems. For example, influenza epidemics have been causing worldwide respiratory deaths ranging from 290,000 to 650,000 each year ${ }^{1}$. The outbreak of coronavirus disease-2019 (COVID-19) caused more than 1 million deaths worldwide ${ }^{2}$. Although vaccination can be an efficient strategy to reduce its hazardous impact, it often takes time to produce an effective vaccine.

Another strategy can be a real-time monitoring system which can detect and alarm respiratory viruses in the air before entering human bodies. Extensive efforts have been given to detect respiratory viruses in the air ${ }^{3-11}$. For example, several groups successfully trapped influenza virus in the air and measured its amount using a quantitative polymerase chain reaction (qPCR) method with a high sensitivity and selectivity ${ }^{3-6}$. However, the qPCR-based method takes some time to get a detection result, and it is not suitable for fast monitoring systems. In other works, nanobiosensors such as silicon nanowire-based biosensors have been utilized to detect influenza viruses captured from the air $^{8,9}$. However, nanobiosensors are often very expensive. Furthermore, the detection limit of nanobiosensors is usually a few PFU level, which may not be enough to detect airborne viruses with a concentration of its threshold infectious dose in the air.

Herein, we report a reusable surface-amplified nanobiosensor for monitoring airborne viruses with a sub$\mathrm{PFU} / \mathrm{mL}$ level detection limit. In this method, antibody-functionalized magnetic particles were utilized to form sandwich structures with target viruses and alkaline phosphatase (ALP) enzyme, and they were trapped on the

\footnotetext{
${ }^{1}$ Department of Physics and Astronomy, Seoul National University, Seoul 08826, Korea. ${ }^{2}$ Department of Physics and Astronomy, and Institute of Applied Physics, Seoul National University, Seoul 08826, Korea. ${ }^{3}$ Gas Metrology Group, Korea Research Institute of Standards and Science (KRISS), Daejeon 34113, Korea. ${ }^{4}$ School of Mechanical Engineering, Yonsei University, Seoul 03722, Korea. ${ }^{5}$ BioNano Health Guard Research Center (H-GUARD), Daejeon 34141, Korea. ${ }^{6}$ Bionanotechnology Research Center, Korea Research Institute of Bioscience and Biotechnology 10 (KRIBB), Daejeon 34141, Korea. ${ }^{7}$ Department of Bioengineering, KRIBB School, University of Science and Technology (UST), Daejeon 34141, Korea. ${ }^{8}$ Department of Chemistry, Sungkyunkwan University, Suwon 16419, Korea. ${ }^{凶}$ email: seunghun@snu.ac.kr
} 


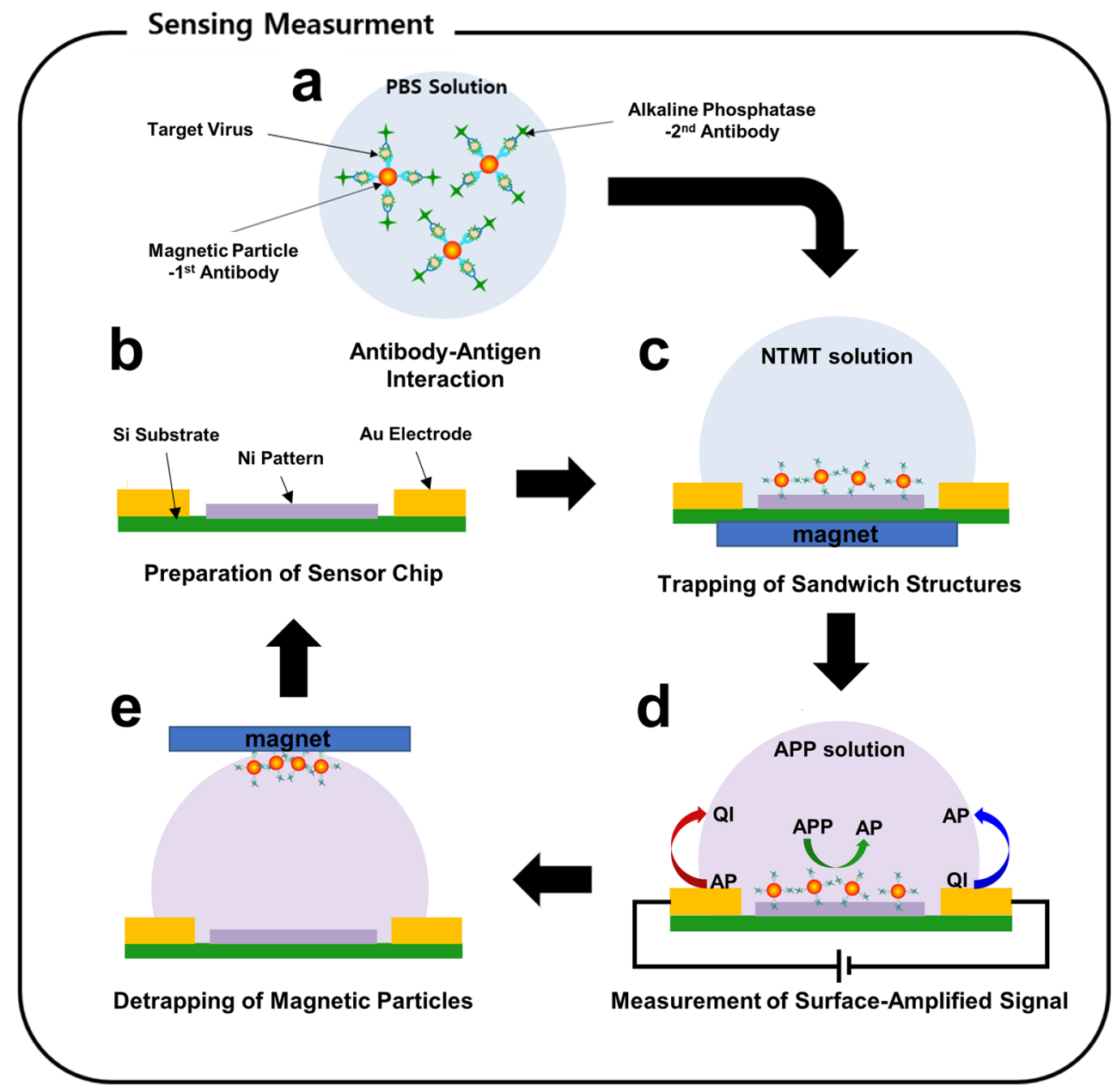

Figure 1. Schematic diagram depicting the cyclic process for the repeated sensing measurements of surfaceamplified sandwich immunoassay. (a) Preparation of sandwich structures in PBS solution including magnetic particles conjugated with 1st antibody, alkaline phosphatase (ALP) conjugated with 2nd antibody, and influenza viruses. (b) Fabrication of a reusable sensor transducer chip including an interdigitated gap sensor and ferromagnetic Ni patterns. (c) Trapping of sandwich structures on Ni patterns between Au electrodes via an external magnetic field. (d) Electrochemical sensing measurement of surface-amplified electrical signals. (e) Detrapping of sandwich structures via an external magnetic field with an opposite direction to that for trapping.

Ni patterns near electrochemical sensor transducers. Then, the electrochemical markers generated by trapped ALPs were measured by the underlying sensor for the highly-sensitive detection of the viruses. The used sandwich structures could be removed via an external magnetic field, enabling repeated sensing measurements. As a proof of concepts, we demonstrated the repeated detection of airborne influenza virus using a single sensor chip with a sub-PFU/mL level detection limit. Our sensor could be utilized repeatedly, for more than 18 times, to measure the hemagglutinin (HA) of influenza $\mathrm{A}(\mathrm{H} 1 \mathrm{~N} 1)$ virus, a widely-used target protein for detecting influenza virus $^{12-15}$, with the concentration ranging from $10 \mathrm{aM}$ to $1 \mathrm{nM}$. Importantly, our sensor chip provided consistent signals even after repeated sensing measurements over the concentration ranges. Moreover, we could utilize our sensor to measure airborne influenza viruses collected from the air with a detection limit down to $0.01 \mathrm{PFU} / \mathrm{mL}$. Interestingly, the detailed quantitative analysis of sensor signals revealed the degradation of HA protein on the virus after the air exposure. Considering the ultra-sensitivity and reusability, our method can be a key stepping stone toward the continuous monitoring system of airborne pathogens, and it can provide a powerful tool for controlling epidemics by preventing airborne pathogens from entering a human body.

\section{Results and discussion}

Figure 1 shows a schematic diagram describing the repeated sensing process of our surface-amplified immunoassay method. Detailed processes are provided in the "Methods" section. Firstly, magnetic particles were functionalized with 1st antibody which selectively binds to HA protein on influenza A (H1N1) virus surface. The schematic diagram of our magnetic particles is presented in supplementary information (Fig. S1). Previous works showed that such magnetic particles maintain their magnetic properties even after the functionalization with biomaterials ${ }^{16,17}$. Alkaline phosphatase (ALP) enzyme was conjugated with its 2 nd antibody. The magnetic 

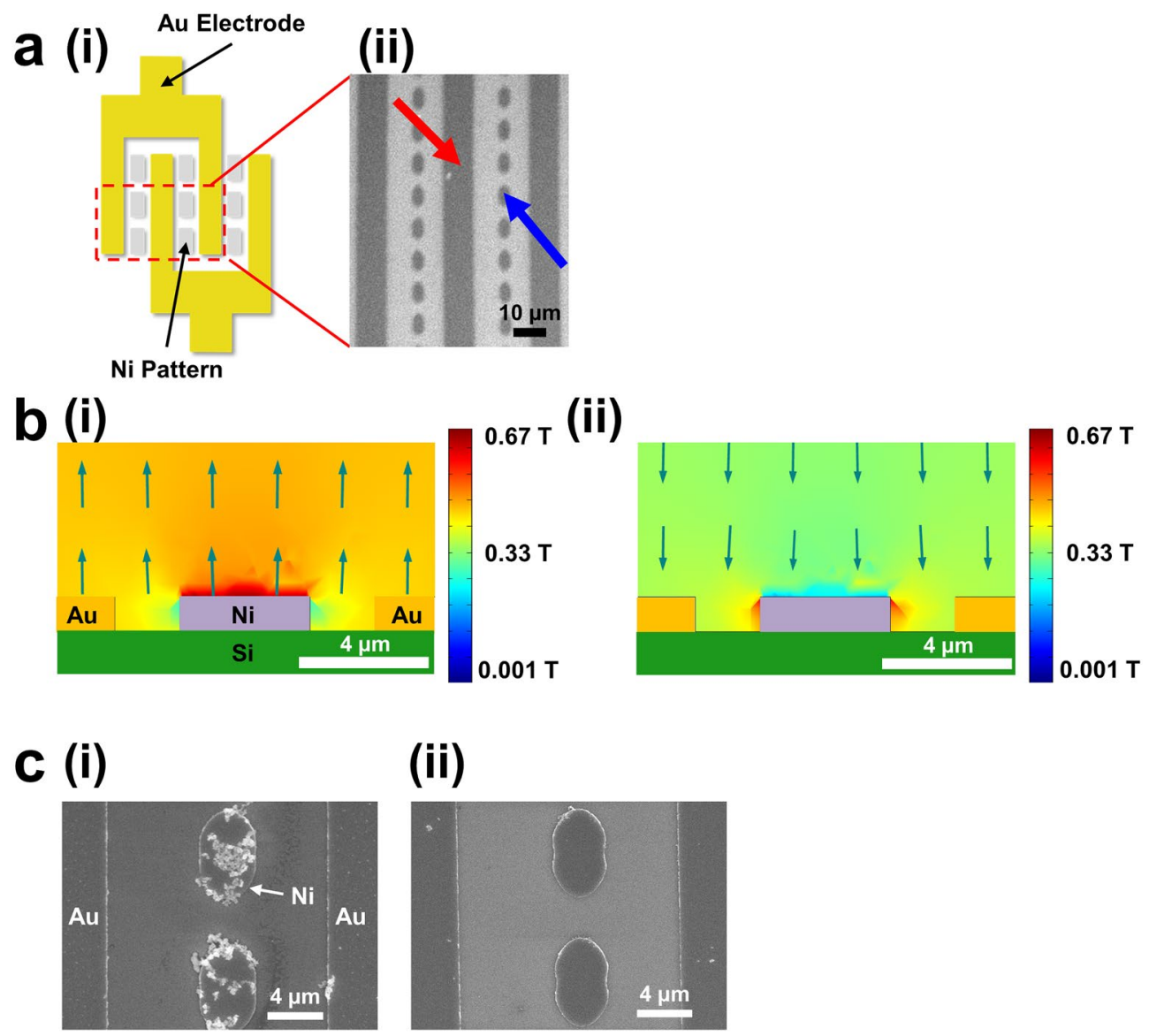

Figure 2. Basic trapping and detrapping operations of our reusable sensor chip. (a) Schematic diagram (i) showing our reusable sensor chip and the field emission scanning electron microscopy (SEM) image (ii) of a sensor chip. (b) Simulation results showing cross-sectional views of a magnetic field strength around a $\mathrm{Ni}$ pattern during trapping (i) and detrapping (ii) processes. (c) SEM images after trapping (i) and detrapping (ii) magnetic particles on Ni patterns.

particles and ALP were mixed with the target solution so that the antibodies bind to the target viruses, resulting in sandwich structures including magnetic particles, influenza virus, and ALP enzyme (Fig. 1a). The solution of the sandwich structures was placed on the fabricated sensor chip including two gold electrodes (90 $\mathrm{nm} \mathrm{Au}$ on $10 \mathrm{~nm} \mathrm{Ti}, 63$ fingers of $10 \mu \mathrm{m}$ width, $30 \mu \mathrm{m}$ gap between the fingers, $2500 \mu \mathrm{m}$ length) and an array of $125 \times 150$ ferromagnetic Ni patterns (10 nm Au on $90 \mathrm{~nm} \mathrm{Ni}, 4 \mu \mathrm{m} \times 8 \mu \mathrm{m}$ ) in-between (Fig. 1b). We used Ni because it has been extensively utilized for practical applications due to some advantageous properties such as a high oxidation resistance and a low fabrication $\operatorname{cost}^{18-20}$. When an external magnetic field was applied, the Ni patterns were magnetized. Then, the patterns attracted magnetic particles in the solution. As a result, the sandwich structures including the influenza virus were concentrated on the ferromagnetic patterns in our sensors (Fig. 1c). 4-aminophenyl phosphate (APP) solution was added to the sandwich structure. Here, ALP enzyme on the Ni patterns generated aminophenols (APs), electrochemical markers, which were measured by using interdigitated Au electrodes with the bias voltages of $+0.1 \mathrm{~V}$ and $-0.1 \mathrm{~V}$ (Fig. 1d). In this method, since a large number of marker molecules were generated by one enzyme molecule in a sandwich structure with only a single virus and many sandwich structures were concentrated on the sensor surface, we could achieve highly-amplified sensor signals. After the sensing process, the used sandwich structures were removed from the sensor chip by applying an external magnetic field in an opposite direction (Fig. 1e). This method allows us to measure extremely low concentration virus solution via signal amplification near the sensor surfaces and to use a single electrode chip for repeated sensing measurements.

Figure 2a shows a schematic diagram (i) and a scanning electron microscopy (SEM) image (ii) of our reusable interdigitated gap sensor chip. In the SEM image, an Au electrode and Ni patterns are marked with red and blue arrows, respectively (Fig. 2a(ii)). The image shows that Ni pattern arrays $(4 \mu \mathrm{m} \times 8 \mu \mathrm{m})$ were fabricated uniformly between interdigitated $\mathrm{Au}$ electrodes. Thus, we can expect that the ferromagnetic patterns could evenly deflect external magnetic fields around the electrode structures so that magnetic particles could be trapped on the patterns without significant aggregations.

Figure $2 \mathrm{~b}$ depicts simulation results showing cross-sectional views of a magnetic field strength around a $\mathrm{Ni}$ pattern during trapping (i) and detrapping (ii) processes. The direction and size of each arrow represent those of 
the magnetic field on that location. For the trapping process, $450 \mathrm{mT}$ of an external magnetic field in an upward direction was applied to a Ni pattern between Au electrodes (Fig. 2b(i)). In this case, the Ni pattern was magnetized along the direction of the field. Due to the magnetic field from the magnetized Ni pattern, a magnetic field strength near the Ni pattern was stronger than those in other regions. For example, the simulation results show that magnetic fields right above the Ni patterns were approximately $600 \mathrm{mT}$, while those of other regions were approximately $450 \mathrm{mT}$ (Fig. $2 \mathrm{~b}$ (i)). Since magnetic particles, due to their magnetic dipole moment, tended to be placed on the strong magnetic field region, they were attracted to the top of Ni patterns and trapped there during a trapping process. Also, note that the strong magnetic field region extended only slightly above the Ni pattern (Fig. 2b). Thus, we can expect that only a thin layer of magnetic particles could be trapped on the Ni patterns without a formation of thick aggregations ${ }^{18,20}$.

For the detrapping process, a rather weak magnetic field $(350 \mathrm{mT})$ in a downward direction opposite to the previously-applied magnetic field was applied to the Ni pattern (Fig. 2b(ii)). However, due to the hysteresis of ferromagnetic Ni patterns, the magnetic polarization of the Ni pattern remained in an upward direction. Since the magnetic fields by the remaining magnetic polarization of the Ni patterns had an opposite direction to that of the external field, the magnetic field strength just above the Ni pattern should be lower than those in other regions. Our simulation results show that the regions above the Ni pattern are darker than other regions, indicating weak magnetic fields. In this case, we can expect that the magnetic particles trapped on the Ni patterns were released from the pattern surface.

Figure 2c (i) and (ii) show the SEM images of Ni patterns after trapping and detrapping of magnetic particles, respectively. Bright particles represented the magnetic particles. The detailed experimental procedure is provided in the "Methods" section. In the trapping process, an external magnetic field of $450 \mathrm{mT}$ was applied normal to the sensor surface, resulting in the selective trapping of magnetic particles only on the Ni patterns (Fig. 2c(i)). Moreover, the trapped particles formed a single layer without forming a large aggregation of magnetic particles.

On the other hand, when a rather weak magnetic field $(350 \mathrm{mT})$ was applied in an opposite direction, magnetic particles were completely removed and, thus, there were no magnetic particles on the Ni patterns (Fig. 2c(ii)). These results show that magnetic nanostructures such as sandwich structures including magnetic particles could be trapped or detrapped on the Ni patterns in our sensor structures, simply by controlling external magnetic fields.

When sandwich structures including magnetic particles and enzymes were trapped on Ni patterns, APP solution was applied so that the enzyme generates 4-Aminophenol (AP) (Fig. 1d). Here, the generated AP, as a electrochemical marker, induced the electrical currents between two electrodes in our sensor surface, which were used as a signal of our sensor (Fig. 3). In this case, the concentration distribution of the generated AP near the sensor surface determines the sensor signals. Figure 3 a depicts the cross-sectional view of numerical simulation results about the concentration distribution of AP generated by the enzyme in the sandwich structures on the $\mathrm{Ni}$ patterns. The yellow rectangles at the bottom of the image represent the Ni patterns. Other regions of the image show the concentration of simulated APs in the buffer solution. In the simulation, $39 \mu \mathrm{L}$ of buffer solution was assumed in a cylinder type well which has a $2.5 \mathrm{~mm}$ radius and $2 \mathrm{~mm}$ height. The $5 \times 20$ array of Ni patterns $(4 \mu \mathrm{m} \times 8 \mu \mathrm{m})$ was placed at the bottom of the well and used to generate APs. We also assumed that the AP was continuously generated on the surfaces of the Ni pattern array and diffused into the buffer solution for $600 \mathrm{~s}$. In the simulation results, the regions near or far from the Ni patterns appeared red or light blue, respectively, indicating that AP concentrations near the Ni patterns were higher than those in regions far from them (Fig. 3a). Note that the AP concentration within $3 \mu \mathrm{m}$ away from the Ni pattern array is 6,400 times higher than the averaged concentration of the whole solution, implying a rather large sensor signal. The simulation results indicate that, in our sensors, AP molecules were generated by the enzyme concentrated near our sensor surface, which enabled large sensor signals and the detection of target molecules with an extremely-high sensitivity.

Figure $3 \mathrm{~b}$ shows typical real-time data of the sensing measurements using hemagglutinin (HA) protein as a target. HA is the protein on influenza $\mathrm{A}(\mathrm{H} 1 \mathrm{N1})$ virus surface and is widely used as a target protein to detect the influenza virus ${ }^{12-15}$. The detailed experimental procedure is presented in the "Methods" section. Briefly, two different solutions of sandwich structures (comprised of magnetic particles, HAs, and ALPs) were prepared using HA solutions with different concentrations of $10 \mathrm{aM}$ and $10 \mathrm{pM}$. Then, HA sensing experiments were performed using the solution with (red or blue lines) or without (black line) trapping the sandwich structures on the Ni patterns of our sensor. Here, the relative conductance change $\left(\Delta G / G_{0}\right)$ of the sensor chip was used as a sensor signal, where $\Delta G$ and $G_{0}$ are the conductance change and original conductance of the sensor chip, respectively. For all experiments in the graph, a single sensor chip was utilized repeatedly. When APP solution was applied to the sandwich structures trapped on the sensor surface, the sensor signal increased sharply and decreased back to a stabilized value at around $600 \mathrm{~s}$ (red and blue lines). The results can be attributed to the redox reaction with aminophenol (AP) and quinone imine (QI) near the cathode and anode electrodes of our sensor. When APP solution was first injected, ALP enzyme in the sandwich structures hydrolyzed most of APP near the sensor surface quickly and generated AP. The generated AP near the cathode electrode were immediately converted to QI by oxidation reactions and provided electrical currents in the electrode, resulting in a sharp increase of sensing signals as reported previously ${ }^{21-23}$. However, after a short time period, most of AP generated from APP was converted to QI, and the sensor signals began to decrease ${ }^{24}$. In this case, the generated QIs diffused to the anode electrode and were converted back to AP by reduction processes. The reduced AP on the anode electrode could diffuse back to the cathode and oxidized again to provide electrical currents ${ }^{25}$. After a while, such oxidation and reduction processes were repeated on cathode and anode electrodes and reached an equilibrium condition, resulting in stable current signals ${ }^{26}$. On the other hand, when the sandwich structures were dispersed in solution without being trapped on the sensor surface, the injected APP was converted to AP slowly over the entire solution. Thus, we could not observe the immediate increase of current signals, and it usually took a rather long time (much longer than $600 \mathrm{~s}$ ) to reach an equilibrium signal (black line in Fig. 3b). Since 
a

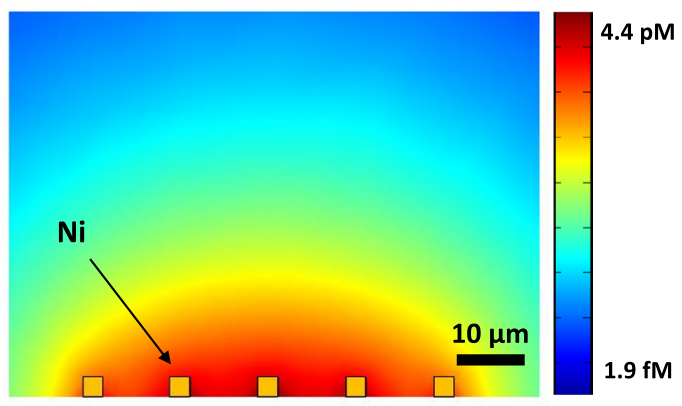

C (i)

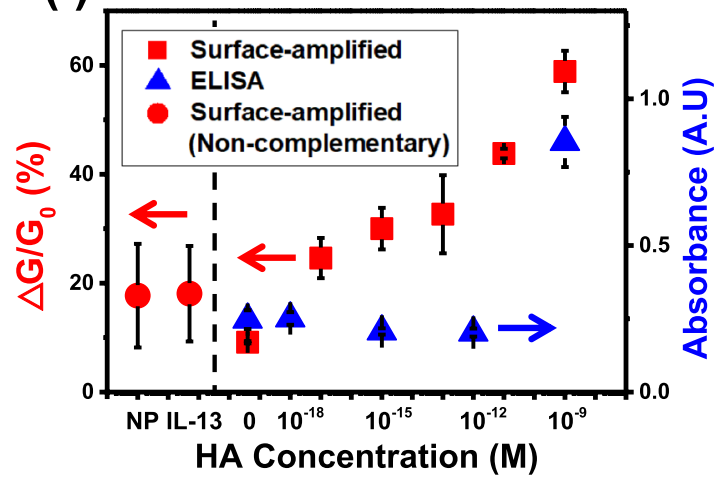

b

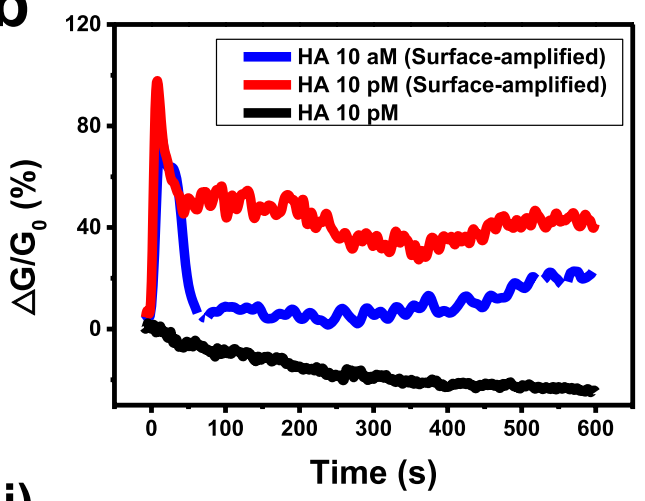

(ii)

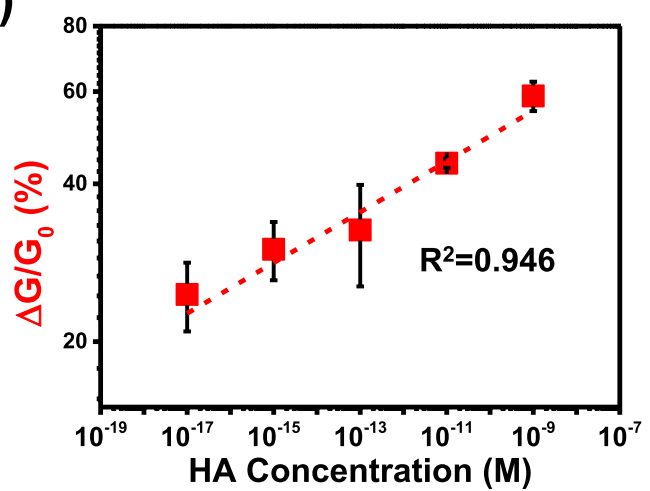

Figure 3. Repeated sensing measurements to detect hemagglutinin (HA) proteins of influenza A (H1N1) virus via surface-amplified sandwich immunoassay. (a) Simulation result showing AP concentrations after AP diffusion in the surface-amplified sandwich immunoassay. (b) Real-time sensor responses to various concentrations of HA with and without the surface amplification of signals. (c) Dose-dependence response curves of the surface-amplified immunoassay $(n=3)$ and conventional ELISA measurements $(n=5)$ using the same 1st and 2nd antibodies (i). Each data point and error bar represents the average value and standard deviation of the experiments. Log-log scale plot showing linearity of our sensor response against HA concentrations (ii).

the signals with trapped sandwich structures were stabilized around $600 \mathrm{~s}$ after the APP injection, the saturated sensor signals were utilized as a sensor signal. Note that the sensor signal for the 10 aM HA solution (blue line) was much smaller than that for the $10 \mathrm{pM} \mathrm{HA}$ solution (red line), indicating that our method can be utilized to quantitatively measure the concentration of target solutions. Also, it should be mentioned that the sensor signal without trapping sandwich structures from 10 pM HA solution (black line) was even smaller than that with trapped sandwich structures from $10 \mathrm{aM}$ solution (blue line), indicating the signal enhancement effect by more than $10^{6}$ times. Presumably, the enzyme in the sandwich structures trapped near the electrodes generated AP with a high concentration near our sensor surface, enhancing the sensor signals. These results show that our strategy of trapping the enzyme-functionalized sandwich structures on the sensor surface can significantly improve the sensor sensitivity.

Figure $3 c$ (i) shows the dose-dependent responses of the HA sensing measurements with our surface-amplified immunoassay strategy (red squares, $\mathrm{n}=3$ ) as well as conventional enzyme-linked immunosorbent assay (ELISA) methods (blue triangles, $\mathrm{n}=5$ ). Here, HA solutions with their concentrations ranging from 10 aM to $1 \mathrm{nM}$ were used for the surface-amplified immunoassay. Also, non-specific responses of our sensor were tested by measuring the sensor responses to non-targeted nucleoproteins (NP) of influenza A (H1N1) virus and interleukin 13 (IL13) with a $10 \mathrm{pM}$ concentration (red circles). It should be noted that all 24 sensing measurements of our surface amplified immunoassay were performed on a single sensor chip (Fig. 3c(i)). Conventional ELISA measurements were performed using the same antibody and HA target protein as our sensors. The detailed experimental procedures are presented in supplementary information. Our sensor began to show responses to 10 aM HA solution, and the responses increased with increasing HA concentrations. However, the non-specific responses to NP and IL-13 were negligibly small compared to those to HA sensing results. These results clearly show the high sensitivity and selectivity of our surface amplified immunoassay. On the other hand, the conventional ELISA method using the same antibody shows sensing signals from the $1 \mathrm{nM} \mathrm{HA}$ solution. Although the same antibodies with their binding constants of a few nanomolar range were used in both sensing measurements, our surface amplified immunoassay showed a detection limit which is lower, by more than $\sim 10^{7}$ times, than that of the conventional ELISA. Presumably, the signal amplification by enzyme near the sensor surface should have enabled such signal enhancements as discussed in Fig. 3b. Importantly, in this sensing experiment, a single sensor was utilized to perform more than 20 times of repeated measurements, while still providing consistent results at 
each concentration of HA (red dots and squares in Fig. 3c(i)). The results show that our sensor can be utilized repeatedly for the detection of hazardous pathogens with a high sensitivity and selectivity.

For a detailed analysis about our sensor responses, we performed a numerical simulation and a fitting for the measured sensor signals (Fig. 3c(ii)). Previous works show that sandwich structure-based biosensors like our sensors exhibited a linear response for different target concentrations ${ }^{27-31}$. Also, a numerical analysis was performed to confirm the linear responses of our sensor. Here, we first set-up differential rate equations regarding individual antibody-antigen bindings during the formation process of sandwich structures and performed a numerical simulation to calculate the sensor signals for different target concentrations (Fig. S2 in supplementary information). The results show that our sensor based on sandwich structures should exhibit a linear response at a rather low target concentration condition. Accordingly, the measured sensor response data were fitted by a linear fitting curve via the linear regression analysis, showing a good fitting result with $\mathrm{R}^{2}=0.946$ (red dashed line in Fig. 3c(ii)). These results show that our sensors can be used to measure a target concentration in a quantitative manner. Furthermore, it also should be mentioned that the sensor exhibited a linear response over a very wide range of target concentrations of $10 \mathrm{aM} \sim 1 \mathrm{nM}$. For various practical applications, a wide analytical range is usually advantageous. However, such a wide analytical range can also limit the resolution of a sensor, which can be disadvantageous for some applications requiring a precise quantitative analysis. For example, a two-fold increase of target concentrations can change our sensor signals only by $\sim 25 \%$. Similar behavior has been reported previously for other biosensors based on antibody bindings ${ }^{32,33}$.

As a proof of concept, our sensor was utilized for the detection of airborne influenza viruses (Fig. 4). Figure 4a shows the schematic diagrams of the experimental set-ups for the aerosolization and collection of influenza viruses. In an atomizer, a solution of influenza viruses was released into the air as aerosols with a flow rate of 2 L/min (Fig. $4 \mathrm{a}(\mathrm{i})$ ). Then, the aerosolized viruses were diluted with clean air to $100 \mathrm{~L} / \mathrm{min}$ in a dilutor (Fig. $4 \mathrm{a}(\mathrm{ii})$ ). Finally, a lab-made electrostatic air sampler was used for sampling the airborne influenza viruses (Fig. 4a(iii)). In the air sampler, the airborne viruses were charged by air ions generated from the tungsten electrode wires via corona discharge. The charged viruses were attracted toward the ground electrode covered with PBS solution, and they were captured in the PBS solution ${ }^{34}$. The number of collected viruses in the solution could be estimated via a conventional analysis method based on a particle counter as shown in the "Methods" section. The solutions of collected viruses were used as a target solution for the sensing measurement of our sensors without any further treatments.

Figure $4 \mathrm{~b}$ displays the dose-dependent responses of our surface-amplified immunoassay using the target solutions of influenza virus before (hollow) and after (red) the aerosolization and collection processes described in Fig. 4a. Here, a single sensor chip was utilized to perform all sensing measurements for both virus solutions with different concentrations ranging from $10^{-2} \mathrm{PFU} / \mathrm{mL}$ to $10^{6} \mathrm{PFU} / \mathrm{mL}$. The same set of measurements were performed repeatedly by three sensor chips to obtain error bars for the data. For both viruses before and after the aerosolization step, our sensor began to respond to virus solutions with its concentration of $0.01 \mathrm{PFU} / \mathrm{mL}$ level. The responses also increased linearly as the concentrations of the virus solutions increased. These results clearly show that our sensor can reliably detect airborne influenza viruses with an sub-PFU/mL level sensitivity using a single sensor chip. Considering that conventional immunoassays usually show a detection limit over a few PFU/mL, our method can be a major breakthrough, and it can be utilized for versatile practical applications such as medical screening and biological research ${ }^{35,36}$.

It is also very interesting to note that, even with the same concentrations, the solutions with viruses after an aerosolization step exhibited a sensor signal slightly smaller than those before the step. Figure $4 \mathrm{c}$ shows a more detailed comparison of the dose-dependence responses for influenza virus solutions before and after the sampling processes. The sensing results at the concentration of $0.01,1$, and $100 \mathrm{PFU} / \mathrm{mL}$ were statistically analyzed. The signals from the collected airborne influenza solutions (hollow bars) were, on average, $21.6 \%$ lower than those of the same concentration virus solutions before sampling (red bars). One plausible explanation can be the reduced binding activity of HA protein on the viruses after the aerosolization step. In our experiment, the concentrations of virus solutions were measured by estimating the number of viruses in the solution as shown in the "Methods" section, while our sensor signals rely on the binding activities of the HA proteins as well as the number of viruses. The slightly reduced sensor signals imply that the binding activity of viruses after the aerosolization was reduced, possibly due to the structural deformation of HA proteins on the virus surfaces during the aerosolization and collection processes. This result about a quantitative measurement shows that our method can be a useful tool for basic researches as well as practical applications.

\section{Conclusions}

A reusable surface-amplified nanobiosensor was developed for monitoring airborne viruses with a sub-PFU/ $\mathrm{mL}$ level detection limit. In this strategy, magnetic particles functionalized with antibody molecules were used to form sandwich structures with target viruses and ALP enzyme, and they were magnetically collected on $\mathrm{Ni}$ patterns close to an electrochemical sensor transducer. Then, electrochemical markers generated by ALP enzyme were measured via the electrochemical sensor transducer for the highly-sensitive detection of viruses. The used sensor transducer chip could be reused after removing the trapped sandwich structures via an eternal magnetic field. As a proof of concepts, our sensor chip was utilized to detect airborne influenza virus with a concentration down to a sub-PFU/mL level. A single reusable sensor chip was used repeatedly to measure HA target proteins of influenza A (H1N1) virus down to $10 \mathrm{aM}$ level. Even after 18 times of repeated sensing measurements, the sensor chip showed consistent results. Additionally, influenza virus solutions with different concentrations could be measured down to $0.01 \mathrm{PFU} / \mathrm{mL}$ level. Importantly, a quantitative analysis of our sensor signals revealed the degradation of HA proteins on the viruses after the air exposure. Considering the reusability and ultrasensitivity, 
a(i)

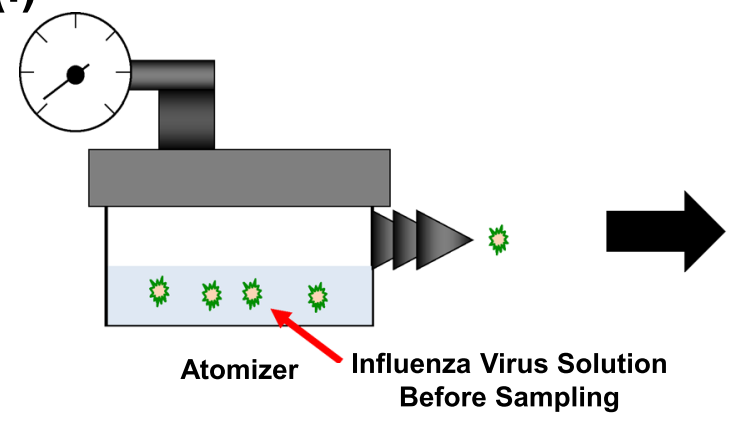

Aerosolization of Influenza Virus Solution (ii)

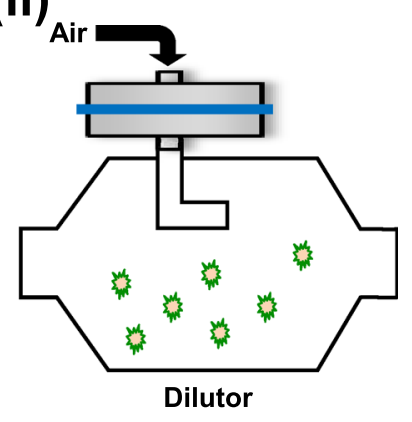

Dilution of Influenza Virus in Air

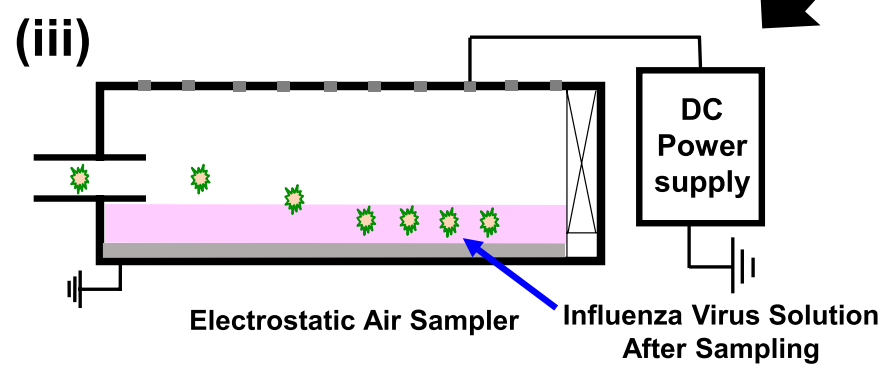

Airborne Influenza Virus Sampling
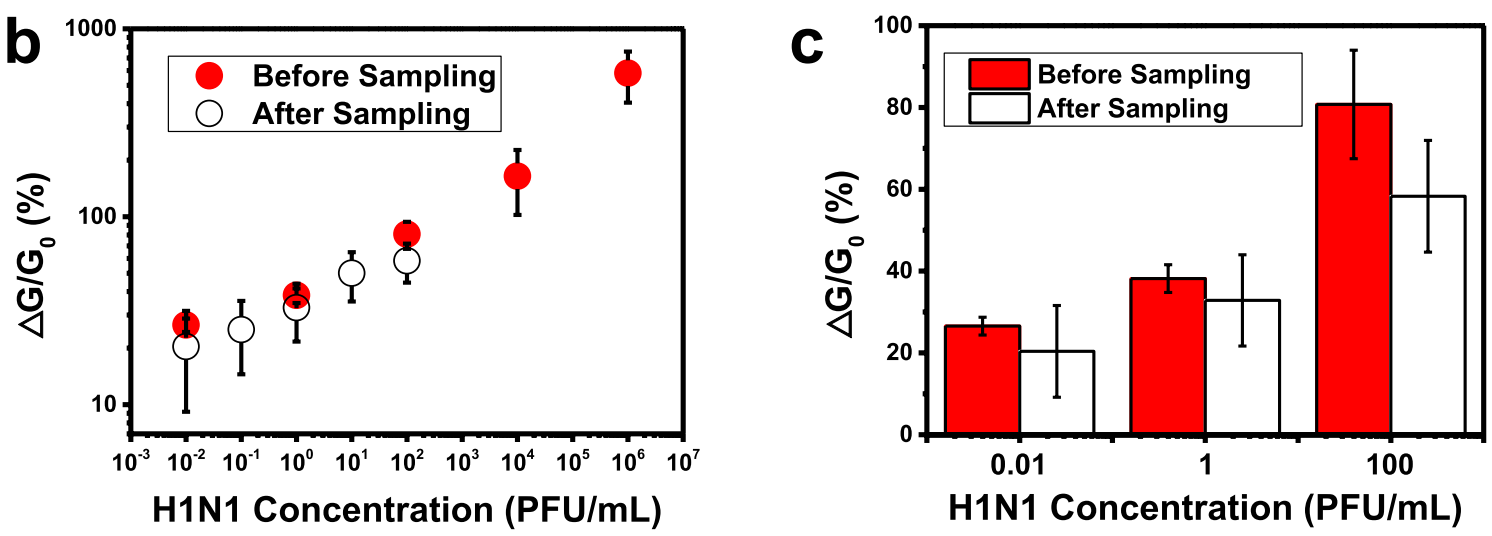

Figure 4. Repeated sensing measurements for the detection of airborne influenza A (H1N1) virus via the surface-amplified sandwich immunoassay. (a) Schematic diagram depicting the aerosolization and collection of influenza virus solution. (i) Aerosolization of influenza virus solution. (ii) Dilution of the aerosolized influenza virus with clean air. (iii) Collection of the aerosolized influenza virus. (b) Dose-dependence response curves of surface-amplified immunoassay to influenza viruses before and after the aerosolization and collection processes. Each data point and error bar represents the average value and standard deviation of the experiments $(n=3)$. $(\mathbf{c})$ Comparison of the sensing measurement results obtained before and after the sampling processes.

our method could be a powerful tool for the monitoring of airborne pathogens and help to prevent epidemics by airborne pathogens.

\section{Methods}

Material. Amine coated superparamagnetic magnetic particles (SiMAG-Amine, with its hydrodynamic diameter of $1 \mu \mathrm{m}, 50 \mathrm{mg} / \mathrm{mL}$ in phosphate buffer saline (PBS) solution) were purchased from Chemicell $\mathrm{GmbH}$ (Germany). Hemagglutinin (HA, A/California/06/2009(H1N1)), anti-HA monoclonal 1st antibody, HRP conjugated anti-HA monoclonal 1st antibody, and anti-HA monoclonal 2nd antibody were obtained from Immune Technology Corp (USA). Influenza A virus solutions (A/California/07/2009 pdmH1N1) were donated by BioNano Health Guard Research Center (H-GUARD). 
Fabrication of reusable sensor transducer chips including interdigitated electrodes and ferromagnetic nickel patterns. Interdigitated $\mathrm{Au}$ gold electrodes $(90 \mathrm{~nm} \mathrm{Au}$ on $10 \mathrm{~nm} \mathrm{Ti}, \mathrm{Ti} / \mathrm{Au}$ ) and ferromagnetic Ni patterns $\left(10 \mathrm{~nm}\right.$ Au on $90 \mathrm{~nm} \mathrm{Ni}$, Ni/Au patterns of size $4 \mu \mathrm{m} \times 8 \mu \mathrm{m}$ ) were fabricated on a $\mathrm{SiO}_{2}$ substrate via conventional photolithography processes.

Trapping and detrapping of magnetic particles on a reusable sensor transducer. Amine coated superparamagnetic magnetic particle solution was diluted to a concentration of $600 \mu \mathrm{g} / \mathrm{mL}$ in PBS solution. Using a pipet, the $20 \mu \mathrm{L}$ droplet of the solution was placed on the sensor transducer chip surface. The $450 \mathrm{mT}$ of an external magnetic field was applied for $1 \mathrm{~min} 30 \mathrm{~s}$ to the sensor chip for trapping the magnetic particles on the Ni patterns. After the trapping process, magnetic particles non-specifically trapped on the surface regions without Ni patterns were removed by pipetting the solution away. For the detrapping of the trapped magnetic particles, the $350 \mathrm{mT}$ of an external magnetic field with an opposite direction was first applied for 2 min to the sensor transducer chip in solution environments. Then, the sensor transducer chip was washed by acetone, ethanol, deionized (DI) water for removing any remaining magnetic particles.

Preparation of magnetic particles functionalized with 1st antibody. Firstly, the anti-HA monoclonal 1st antibody solution was diluted to a $200 \mathrm{nM}$ concentration in $150 \mu \mathrm{L}$ PBS solution. $18 \mu \mathrm{L}$ of Amine coated superparamagnetic magnetic particle solution was prepared. By using a magnetic separator, the magnetic particles were washed 2 times with $200 \mu \mathrm{L}$ of 2-(N-morpholino)ethanesulfonic acid (MES) buffer $(0.1 \mathrm{M}, \mathrm{pH}$ 6.0). The magnetic particles were separated from the solution by a magnet. After removing the supernatant, 300 $\mu \mathrm{L}$ MES buffer was added to the magnetic particles. 1-ethyl-3-(3-dimethylaminopropyl)carbodiimide (EDC) and N-hydroxysuccinimide (NHS) (GE Healthcare, USA) were dissolved in MES buffer. $240 \mu \mathrm{L}$ of the magnetic particle solution was mixed with $60 \mu \mathrm{L}$ of the MES buffer containing $5 \mathrm{mM}$ EDC and $20 \mathrm{mM}$ NHS. $150 \mu \mathrm{L}$ of the solution was added to the 1 st antibody solution. The solution was gently mixed for $2 \mathrm{~h}$ at room temperature. The magnetic particles functionalized with 1st antibody were washed 3 times in $200 \mu \mathrm{L}$ of PBS buffer by using the magnetic separator. The magnetic particles were separated from the solution by a magnet. After removing the supernatant, $300 \mu \mathrm{L}$ PBS buffer was added to the magnetic particles.

Preparation of ALP functionalized with 2nd antibody. An alkaline phosphatase labeling kit was purchased from dojindo molecular technologies (USA). 2nd antibody was conjugated with ALP following the technical manual provided by the vendor.

Sandwich structures including HA protein. $27 \mathrm{~g}$ of blocking reagent for ELISA (Roche, Swiss) was dissolved in $1 \mathrm{~L}$ of PBS buffer. HA protein solution was prepared with the desired concentration in the blocking buffer. $50 \mu \mathrm{L}$ of the prepared magnetic particles functionalized with 1st antibody solution was added to $500 \mu \mathrm{L}$ of the prepared HA solutions. The mixed solution was incubated at $37^{\circ} \mathrm{C}$ for $2 \mathrm{~h}$. The solution of ALP functionalized with 2nd antibody was diluted to $120 \mathrm{pM}$ of 2nd antibody concentration in the blocking buffer. Then, 50 $\mu \mathrm{L}$ of the solution was injected into the mixed solution. The solution was incubated at $37^{\circ} \mathrm{C}$ for $1 \mathrm{~h}$. By using a magnetic separator, the sandwich structure solution was washed 3 times with $200 \mu \mathrm{L}$ of PBS solution. The sandwich structures were separated from the solution by a magnet. After removing the supernatant, $100 \mu \mathrm{L}$ of alkaline phosphatase buffer (NTMT) buffer $(100 \mathrm{mM} \mathrm{NaCl}, 100 \mathrm{mM}$ Tris-Cl (pH 9.0), $50 \mathrm{mM} \mathrm{MgCl}, 1 \%$ tween 20) was added to the sandwich structures.

Influenza virus aerosolization and collection. The detailed experimental setup is presented in Fig. S3. Influenza A (H1N1) virus solution was prepared following procedures in supplementary information. The stocks of influenza viruses were diluted with deionized water to $4.33 \times 10^{5} \mathrm{PFU} / \mathrm{mL}$. The solution was aerosolized using an atomizer (9302, TSI., USA) with a $2 \mathrm{~L} / \mathrm{min}$ flow rate of clean compressed air. A diffusion dryer was used to eliminate moisture of the aerosolized virus solution. Then, the virus particles passed through a neutralizer (Soft X-ray charger 4530, HTC, Korea) to induce a Boltzmann charge distribution. The virus-laden air flow was diluted with clean air to $100 \mathrm{~L} / \mathrm{min}$ and then placed into the air sampler. Airborne viruses entering the air sampler were captured on the ground electrode covered with $\mathrm{PBS}^{34}$.

Quantification of airborne influenza virus collected after aerosolization. The collection efficiency $\left(\eta_{\text {collection }}\right)$ of the air sampler for the test viruses was calculated by the following equation:

$$
\eta_{\text {collection }}=1-\frac{\mathrm{C}_{\mathrm{on}}}{\mathrm{C}_{\mathrm{off}}}
$$

where $C_{o n}$ and $C_{o f f}$ are the number concentrations of aerosolized viruses measured downstream of the sampler with a scanning mobility particle sizer (SMPS, TSI, USA) when the power is on and off, respectively. The virus collection efficiency ( $\eta_{\text {collection }}$ ) of the sampler was $0.7 \pm 0.04$ with the sampling flow rate of $100 \mathrm{~L} / \mathrm{min}$ and the applied voltage of $-10 \mathrm{kV}$ (Fig. S4 in supplementary information). The virus sampling was carried out for $60 \mathrm{~min}$ with an airflow rate of $100 \mathrm{~L} / \mathrm{min}$ and an applied voltage of $-10 \mathrm{kV}$. The concentration of the collected virus sample $\left(\mathrm{C}_{\text {sample }}\right)$ was calculated using the following equation:

$$
\mathrm{C}_{\text {sample }}=\frac{\mathrm{C}_{\text {air }}{ }^{*} \mathrm{Q}^{*} \eta_{\text {collection }}{ }^{*} \mathrm{t}}{\mathrm{V}}
$$


where $\mathrm{C}_{\text {air }}$ is aerosolized virus concentration $\left(4.33 \times 10^{3} \mathrm{PFU} / \mathrm{L}\right), \mathrm{Q}$ is air flow rate of the sampler $(100 \mathrm{~L} / \mathrm{min})$, $\eta_{\text {collection }}$ is the collection efficiency (0.7), $\mathrm{t}$ is virus sampling time $(60 \mathrm{~min})$, and $\mathrm{V}$ is volume of collection medium $(20 \mathrm{~mL})$. Therefore, the concentration of collected virus sample was $910 \mathrm{PFU} / \mathrm{mL}$.

Sandwich structures including influenza viruses. Influenza virus solution with the desired concentration was prepared in the blocking buffer. Sandwich structures were prepared in the same way as in the preparation of sandwich structures including HA, except that influenza virus solution was used for forming sandwich structures instead of HA protein solution.

Repeated sensing operations of reusable interdigitated gap sensors. A cylindrical Polydimethylsiloxane (PDMS, Sylgard ${ }^{\mathrm{m}}$ 184, Sigma-Aldrich) well (5 mm diameter, $5 \mathrm{~mm}$ height) was placed on a reusable interdigitated gap sensor. $20 \mu \mathrm{L}$ of $1 \% \mathrm{w} / \mathrm{v}$ bovine serum albumin (BSA) solution was introduced into the PDMS well. After $5 \mathrm{~min}$, the BSA solution was removed. Then, the $20 \mu \mathrm{L}$ of sandwich structure solution was placed in the well. The $450 \mathrm{mT}$ of an external magnetic field was applied for $1 \mathrm{~min} 30 \mathrm{~s}$ to the sensor chip for trapping the sandwich structures on the Ni patterns. After the trapping process, the sandwich structures trapped on the sensor surfaces without $\mathrm{Ni}$ patterns were removed by pipetting the solution away. $0.2 \mathrm{~V}$ of bias voltage was applied to the $\mathrm{Au}$ electrodes of the sensor chip. The current of the sensor chip was measured with a bipotentiostat $(\mu$ Stat 400 , DropSens). After the stabilization of the current signals, the stabilized current signals were recorded as baseline signals. Then, $20 \mu \mathrm{L}$ of $10 \mathrm{mM}$ APP solution in NTMT buffer was added to the sandwich structure solution. After $10 \mathrm{~min}$, the current signals were recorded as sensing signals. When a sensing measurement was completed, the $350 \mathrm{mT}$ of an external magnetic field was applied for $2 \mathrm{~min}$ to the sensor chip for detrapping the sandwich structures. The sensor chip was washed with acetone, ethanol, and DI water for removing the remained magnetic particles. The sensor chip was irradiated with ultraviolet (UV) light ( $278 \mathrm{~nm}$ wavelength) for UV/ozone surface cleaning.

Received: 14 May 2021; Accepted: 6 August 2021

Published online: 18 August 2021

\section{References}

1. Du, Z. W., Nugent, C., Galvani, A. P., Krug, R. M. \& Meyers, L. A. Modeling mitigation of influenza epidemics by baloxavir. Nat. Commun. 11, 2750 (2020).

2. Ioannidis, J. P. A. Global perspective of COVID-19 epidemiology for a full-cycle pandemic. Eur. J. Clin. Invest. 50, e13423 (2020).

3. Ladhani, L. et al. Sampling and detection of airborne influenza virus towards point-of-care applications. PLoS ONE 12, e0174314 (2017).

4. Pyankov, O. V. et al. Using a bioaerosol personal sampler in combination with real-time PCR analysis for rapid detection of airborne viruses. Environ. Microbiol. 9, 992-1000 (2007).

5. Kim, H. R., An, S. \& Hwang, J. An integrated system of air sampling and simultaneous enrichment for rapid biosensing of airborne coronavirus and influenza virus. Biosens. Bioelectron. 170, 112656 (2020).

6. McDevitt, J. J. et al. Development and performance evaluation of an exhaled-breath bioaerosol collector for influenza virus. Aerosol. Sci. Tech. 47, 444-451 (2013).

7. Kwon, H. J., Fronczek, C. F., Angus, S. V., Nicolini, A. M. \& Yoon, J. Y. Rapid and sensitive detection of H1N1/2009 virus from aerosol samples with a microfluidic immunosensor. Jala-J. Lab. Autom. 19, 322-331 (2014).

8. Shen, F. X. et al. Integrating silicon nanowire field effect transistor, microfluidics and air sampling techniques for real-time monitoring biological aerosols. Environ. Sci. Technol. 45, 7473-7480 (2011).

9. Shen, F. X. et al. Rapid flu diagnosis using silicon nanowire sensor. Nano Lett. 12, 3722-3730 (2012).

10. Takenaka, K., Togashi, S., Miyake, R., Sakaguchi, T. \& Hide, M. Airborne virus detection by a sensing system using a disposable integrated impaction device. J. Breath. Res. 10, 036009 (2016).

11. Usachev, E. V., Agranovski, E., Usacheva, O. V. \& Agranovski, I. E. Multiplexed surface plasmon resonance based real time viral aerosol detection. J. Aerosol. Sci. 90, 136-143 (2015).

12. Matsubara, T. et al. Avian influenza virus detection by optimized peptide termination on a boron-doped diamond electrode. Acs Sens. 5, 431-439 (2020).

13. Egashira, N., Morita, S., Hifumi, E., Mitoma, Y. \& Uda, T. Attomole detection of hemagglutinin molecule of influenza virus by combining an electrochemiluminescence sensor with an immunoliposome that encapsulates a Ru complex. Anal. Chem. 80, 4020-4025 (2008).

14. Horiguchi, Y. et al. Direct and label-free influenza virus detection based on multisite binding to sialic acid receptors. Biosens. Bioelectron. 92, 234-240 (2017).

15. Takemura, K. et al. Versatility of a localized surface plasmon resonance-based gold nanoparticle-alloyed quantum dot nanobiosensor for immunofluorescence detection of viruses. Biosens. Bioelectron. 89, 998-1005 (2017).

16. Adolphi, N. L. et al. Characterization of magnetite nanoparticles for SQUID-relaxometry and magnetic needle biopsy. J. Magn. Magn. Mater. 321, 1459-1464 (2009).

17. Flynn, E. R. et al. Use of a SQUID array to detect T-cells with magnetic nanoparticles in determining transplant rejection. J. Magn. Magn. Mater. 311, 429-435 (2007).

18. Yoo, H. et al. Magnetically-refreshable receptor platform structures for reusable nano-biosensor chips. Nanotechnology 27, 045502 (2016).

19. Yoo, H. et al. Magnetically-focusing biochip structures for high-speed active biosensing with improved selectivity. Nanotechnology 29, 265501 (2018).

20. Yoo, H., Shin, J., Sim, J., Cho, H. \& Hong, S. Reusable surface plasmon resonance biosensor chip for the detection of H1N1 influenza virus. Biosens. Bioelectron. 168, 112561 (2020).

21. Fiaschi, G. et al. A novel gas-phase mono and bimetallic clusters decorated Zno nanorods electrochemical sensor for 4-aminophenol detection. J. Electroanal. Chem. 811, 89-95 (2018).

22. Sen, M. et al. LSI-based amperometric sensor for real-time monitoring of embryoid bodies. Biosens. Bioelectron. 48, 12-18 (2013). 
23. Serra, B., Morales, M. D., Reviejo, A. J., Hall, E. H. \& Pingarron, J. M. Rapid and highly sensitive electrochemical determination of alkaline phosphatase using a composite tyrosinase biosensor. Anal. Biochem. 336, 289-294 (2005).

24. Nassef, H. M., Radi, A. E. \& O'Sullivan, C. Simultaneous detection of ascorbate and uric acid using a selectively catalytic surface. Anal. Chim. Acta 583, 182-189 (2007).

25. Neugebauer, S., Stoica, L., Guschin, D. \& Schuhmann, W. Redox-amplified biosensors based on selective modification of nanopore electrode structures with enzymes entrapped within electrodeposition paints. Microchim. Acta 163, 33-40 (2008).

26. Thomas, J. H., Kim, S. K., Hesketh, P. J., Halsall, H. B. \& Heineman, W. R. Microbead-based electrochemical immunoassay with interdigitated array electrodes. Anal. Biochem. 328, 113-122 (2004).

27. Gaster, R. S. et al. Matrix-insensitive protein assays push the limits of biosensors in medicine. Nat. Med. 15, 1327-U1130 (2009).

28. Zhu, L., Li, G. H., Sun, S. Q., Tan, H. \& He, Y. H. Digital immunoassay of a prostate-specific antigen using gold nanorods and magnetic nanoparticles. Rsc Adv. 7, 27595-27602 (2017).

29. Kim, W. J. et al. Highly sensitive detection of cardiac troponin I in human serum using gold nanoparticle-based enhanced sandwich immunoassay. Sens. Actuat. B-Chem. 221, 537-543 (2015).

30. Warren, A. D. et al. Disease detection by ultrasensitive quantification of microdosed synthetic urinary biomarkers. J. Am. Chem. Soc. 136, 13709-13714 (2014).

31. Ma, X. Y. et al. SERS aptasensor detection of Salmonella typhimurium using a magnetic gold nanoparticle and gold nanoparticle based sandwich structure. Anal. Methods-Uk 8, 8099-8105 (2016).

32. Coudron, L. et al. Fully integrated digital microfluidics platform for automated immunoassay; A versatile tool for rapid, specific detection of a wide range of pathogens. Biosens. Bioelectron. 128, 52-60 (2019).

33. Lai, G. S., Zhang, H. L., Tamanna, T. \& Yu, A. M. Ultrasensitive immunoassay based on electrochemical measurement of enzymatically produced polyaniline. Anal. Chem. 86, 1789-1793 (2014).

34. Hyeong Rae Kim, S. A. \& Hwang, J. High air flow-rate electrostatic sampler for the rapid monitoring of airborne coronavirus and influenza viruses. J. Hazard. Mater. 412, 125219 (2021).

35. Cheng, M. S. et al. Development of an electrochemical membrane-based nanobiosensor for ultrasensitive detection of dengue virus. Anal. Chim. Acta 725, 74-80 (2012).

36. Oh, S. et al. Magnetic nanozyme-linked immunosorbent assay for ultrasensitive influenza a virus detection. Acs Appl. Mater. Int. 10, 12534-12543 (2018).

\section{Acknowledgements}

This work was supported by the National Research Foundation of Korea (NRF) funded by the Ministry of Science and ICT (MSIT) of Korea (No. 2013M3A6B2078961, 2013M3A6B2078950 and 2020R1A2B5B02002152). S.H. would like to acknowledge the support from the Ministry of Trade, Industry \& Energy (MOTIE, Korea) (20012390), Samsung Electronics Co. Ltd. (No. 201209-07908-01) and the European Research Council (ERC) under the European Union's Horizon 2020 programme (Grant Agreement No. 682286). J.H. would like to acknowledge the support from the Korea Environment Industry \& Technology Institute (KEITI) through Technology Development Project for Biological Hazards Management in Indoor Air Program funded by Korea Ministry of Environment (MOE) (G232021010381).

\section{Author contributions}

The key idea was conceived by J.H., S.H., A.K., and W.S.Y. J.S. designed the study, performed most experiments, analyzed the data, and wrote the manuscript. H.R.K. aerosolized and collected the virus. P.K.B. prepared the influenza virus solution. H.Y., J.K., and Y.C. wrote the paper. Y.S. prepared the influenza virus solution. J.H. aerosolized and collected the virus. S.H. is responsible for the project and contributed to data analyses. The manuscript was written through the contributions of all authors. All authors have given approval to the final version of the manuscript.

\section{Competing interests}

The authors declare no competing interests.

\section{Additional information}

Supplementary Information The online version contains supplementary material available at https://doi.org/ 10.1038/s41598-021-96254-2.

Correspondence and requests for materials should be addressed to S.H.

Reprints and permissions information is available at www.nature.com/reprints.

Publisher's note Springer Nature remains neutral with regard to jurisdictional claims in published maps and institutional affiliations.

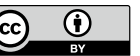

Open Access This article is licensed under a Creative Commons Attribution 4.0 International License, which permits use, sharing, adaptation, distribution and reproduction in any medium or format, as long as you give appropriate credit to the original author(s) and the source, provide a link to the Creative Commons licence, and indicate if changes were made. The images or other third party material in this article are included in the article's Creative Commons licence, unless indicated otherwise in a credit line to the material. If material is not included in the article's Creative Commons licence and your intended use is not permitted by statutory regulation or exceeds the permitted use, you will need to obtain permission directly from the copyright holder. To view a copy of this licence, visit http://creativecommons.org/licenses/by/4.0/.

(C) The Author(s) 2021, corrected publication 2021 